Gerión. Revista de Historia Antigua

ISSN: 0213-0181

\title{
Sobre la capacidad de almacenaje de los horrea rurales romanos: teoría del Escepticismo Cuantitativo
}

\author{
Javier Salido Domínguez ${ }^{1}$
}

Recibido: 7 de febrero de 2017 / Aceptado: 22 de marzo de 2018

Resumen. Se presenta un balance sobre la información que nos aportan los análisis arqueobotánicos para el estudio del almacenaje de cereal en época romana, que requería de unas condiciones óptimas de temperatura y humedad para su conservación. El objetivo de este artículo es cuestionar algunas propuestas de capacidad de los horrea romanos indicando los múltiples factores que condicionan no sólo la construcción de los graneros, sino también la organización y disposición del cereal almacenado. En definitiva, tratamos de plantear la Teoría del Escepticismo Cuantitativo (TEC) con el fin de abordar la problemática de los cálculos de capacidad de almacenaje desde un punto de vista arqueológico.

Palabras clave: economía romana; arqueobotánica; cereal; almacenaje; horrea; capacidad.

\section{[en] On the storage capacity of the Roman rural horrea: Theory of Quantitative Skepticism}

\begin{abstract}
We present the information provided by archaeobotanical analysis for the study of cereal storage in Roman times that required optimal conditions of temperature and humidity for its preservation. The aim of this article is to question some proposals on the capacity of the Roman horrea indicating the multiple factors that determine not only their construction, but also the organization and disposition of the stored cereal. In short, we try to raise the Theory of Quantitative Skepticism (TEC) in order to address the problem of calculations of storage capacity from an archaeological point of view.

Keywords: Roman economy; Archaeobotany; Cereals; Storage; horrea; Capacity.
\end{abstract}

Sumario. 1. Introducción. 2. El medio ambiente: los estudios palinológicos aplicados al estudio de los horrea. 3. ¿Qué especies de grano se almacenaron en los horrea rurales romanos? 4. No sólo grano: otros alimentos almacenados en los horrea. 5. ¿Cómo se almacenó el cereal? 6. Las consecuencias de una mala conservación o un almacenaje prolongado: la germinación. 7. La Teoría del Escepticismo Cuantitativo (TEC). 8. Referencias bibliográficas.

Cómo citar: Salido Domínguez, J. (2018): Sobre la capacidad de almacenaje de los horrea rurales romanos: teoría del Escepticismo Cuantitativo, en Gerión 36/1, 79-94.

\footnotetext{
$1 \quad$ Universidad Complutense de Madrid.

E-mail: pjaviers@hotmail.com
} 


\section{Introducción}

En los últimos años se ha puesto de relieve la importancia del análisis de los graneros y horrea romanos con el fin de reconocer a nivel arqueológico la vocación agrícola cerealista de las villae romanas. ${ }^{2}$ La aplicación de los análisis arqueobotánicos, dentro de investigaciones interdisciplinares e incluidos en los planes de excavación, se alzan hoy en día como uno de los instrumentos necesarios para el conocimiento del medio vegetal y de los productos almacenados en los graneros romanos. Aunque cada vez se van dedicando más esfuerzos en el estudio de la producción y almacenaje de alimentos a partir de las muestras recogidas, todavía es preciso aumentar nuestra voluntad para aproximarnos al análisis de la economía romana con este tipo de herramientas.

Respecto al almacenaje de grano en los horrea, actualmente existe un debate bastante controvertido sobre la capacidad de los almacenes. No cabe duda de que es una cuestión importante, porque su estimación aproximada nos permitiría llegar a conclusiones interesantes sobre la gestión y el abastecimiento del cereal, determinando si los granaria rurales son almacenes de capacidad limitada para los trabajadores y propietarios de las villae o si, además, funcionan como verdaderos depósitos que permiten abastecer a otras poblaciones en épocas de carestía. Sobre esta problemática, Gentry y Manning ${ }^{3}$ ya plantearon unos cálculos generales de capacidad de los graneros que, con criterios diferentes, pretendían establecer los parámetros para medir la capacidad de un horreum con el fin de explicar la información aportada por Tácito, ${ }^{4}$ quien señaló que estos almacenes militares tenían capacidad para guardar el grano de la unidad militar acantonada durante un año. Desde la publicación de estos trabajos, han sido múltiples las propuestas planteadas por los diferentes investigadores, según el tipo y el peso del grano establecido, la altura del amontonamiento, el modo de almacenaje, etc. Entre estas teorías, destacan las propuestas de los grandes horrea civiles de Ostia ${ }^{5}$ y ya indicamos en trabajos anteriores las referidas a los horrea militares ${ }^{6}$ y rurales. ${ }^{7}$ Respecto a estos últimos, destacan las propuestas sobre la capacidad de los horrea de Biberist-Spitalhof (Wasseramt, Solothurn, Suiza), ${ }^{8}$ Bois du Châtel en Vieux-Champagne (Île-de-France, Francia), ${ }^{9}$ Houten-Tielland (Utrecht, Países Bajos) ${ }^{10}$ y Rockanje (Zuid-Holland, Países Bajos (Fig. 1). ${ }^{11}$

En este trabajo nos proponemos destacar los factores que nos permiten cuestionar gran parte de los cálculos propuestos, bajo la denominación de la Teoría del Escepticismo Cuantitativo. La mayor parte de la información que nos permite dudar sobre las propuestas de capacidad proceden de los resultados de los análisis arqueobotánicos, como iremos analizando a continuación.

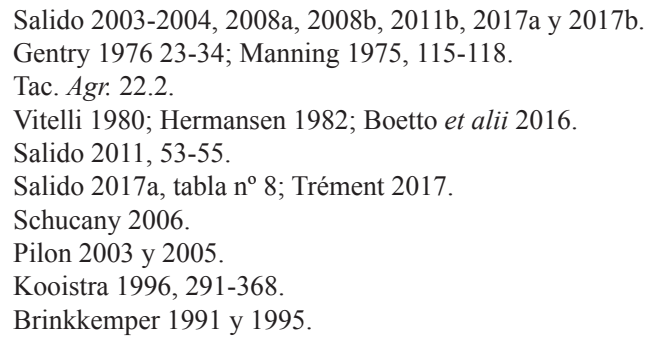




\begin{tabular}{|c|c|c|c|c|c|}
\hline Yacimiento & $\begin{array}{l}\text { Tipo de } \\
\text { granero }\end{array}$ & $\begin{array}{c}\text { Superficie de } \\
\text { la cámara de } \\
\text { almacenaje }\left(\mathbf{m}^{2}\right)\end{array}$ & $\begin{array}{l}\text { Capacidad } \\
\qquad\left(\mathbf{m}^{3}\right)\end{array}$ & Peso (tn) & $\begin{array}{l}\text { Altura del cereal } \\
\text { almacenado }\end{array}$ \\
\hline $\begin{array}{l}\text { Biberist-Spitalhof } \\
\text { (Suiza) }\end{array}$ & $\begin{array}{l}\text { Sobre } \\
\text { postes }\end{array}$ & $36 \mathrm{~m}^{2}$ & $100 \mathrm{~m}^{3}$ & $78 \mathrm{tn}$ & $\begin{array}{l}2,77 \text { m según } \\
\text { la propuesta de } \\
\text { Gentry }\end{array}$ \\
\hline $\begin{array}{c}\text { Vieux- } \\
\text { Champagne, } \\
\text { Bois du Châtel } \\
\text { (Francia) }\end{array}$ & $\begin{array}{l}\text { Sobre } \\
\text { muros de } \\
\text { piedra }\end{array}$ & & $24 \mathrm{~m}^{3}$ & $20 \mathrm{tn}$ & \\
\hline $\begin{array}{l}\text { Houten-Tielland } \\
\text { (Utrecht, Países } \\
\text { Bajos)- granero } 1\end{array}$ & $\begin{array}{l}\text { Sobre } \\
\text { postes }\end{array}$ & $26,1 \mathrm{~m}^{2}$ & $16 \mathrm{~m}^{3}$ & $\begin{array}{c}\text { No } \\
\text { disponible }\end{array}$ & $\begin{array}{c}0,61 \text { m según } \\
\text { la propuesta de } \\
\text { Gentry }\end{array}$ \\
\hline $\begin{array}{l}\text { Houten-Tielland } \\
\text { (Utrecht, Países } \\
\text { Bajos)- granero } 2\end{array}$ & $\begin{array}{l}\text { Sobre } \\
\text { postes }\end{array}$ & $66,95 \mathrm{~m}^{2}$ & $40 \mathrm{~m}^{3}$ & $\begin{array}{c}\text { No } \\
\text { disponible }\end{array}$ & $\begin{array}{l}0,59 \text { m según } \\
\text { la propuesta de } \\
\text { Gentry }\end{array}$ \\
\hline $\begin{array}{c}\text { Rockanje } \\
\text { (Zuid-Holland, } \\
\text { Países Bajos) }\end{array}$ & $\begin{array}{l}\text { Sobre } \\
\text { postes }\end{array}$ & $12,6 \mathrm{~m}^{2}$ & $4,8 \mathrm{~m}^{3}$ & 4 tn & $\begin{array}{c}1,15 \mathrm{~m} \\
0,38 \mathrm{~m} \text { según } \\
\text { la propuesta de } \\
\text { Gentry }\end{array}$ \\
\hline
\end{tabular}

Figura 1. Tabla comparativa sobre las principales propuestas de capacidad de almacenaje de los graneros rurales romanos.

\section{El medio ambiente: los estudios palinológicos aplicados al estudio de los horrea}

En algunos casos son los análisis palinológicos los únicos que nos aportan datos sobre el tipo de producción agrícola del entorno donde se encuentra el yacimiento. Es el caso del horreum de Veranes (Gijón) ${ }^{12}$ que, cuando sirvió de granero en época altoimperial, se integraba en un paisaje enormemente deforestado, casi sin árboles, en el que apenas restaban rodales muy dispersos de especies caducifolias -principalmente de robles (Quercus robur tipo), acompañados de alisos (Alnus), abedules (Betula), fresnos (Fraxinus), avellanos (Corylus) y álamos (Populus)entre los cuales se intercalarían, de manera diseminada, elementos arbustivos propios de matorrales degradativos del bosque, como enebros (Juniperus tipo), brezos (Erica arborea tipo) o tojos (Cytisus tipo); e incluso se empezarían a plantar los primeros nogales ${ }^{13}$ (Figs. 2 y 3). En cambio, en fechas avanzadas del siglo IV d.C., cuando el edificio cambió de función, pasando a ser un almacén o despensa, el paisaje comenzó a hacerse más cerrado, con predominio del bosque caducifolio de robles y avellanos, alisos y álamos, así como del bosque con diversas especies de pino, entre las que destaca el pino resinero (Pinus pinaster), manteniéndose el

Fernández Ochoa et alii 2012.

López Sáez 2012, 168. 


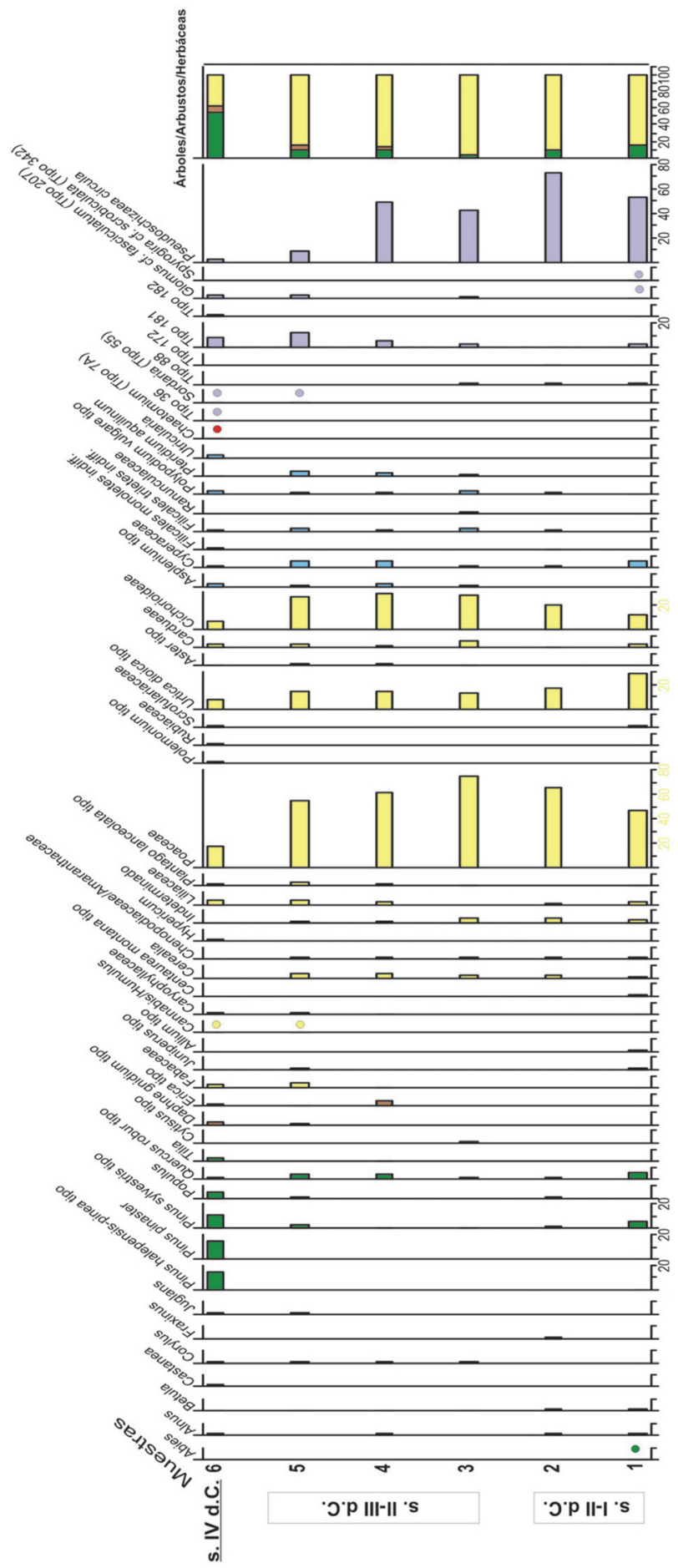

Figura 2. Resultados palinológicos del granero de la villa romana de Veranes (Gijón). Muestra E5 (López Sáez 2012, 163, fig. 74). 


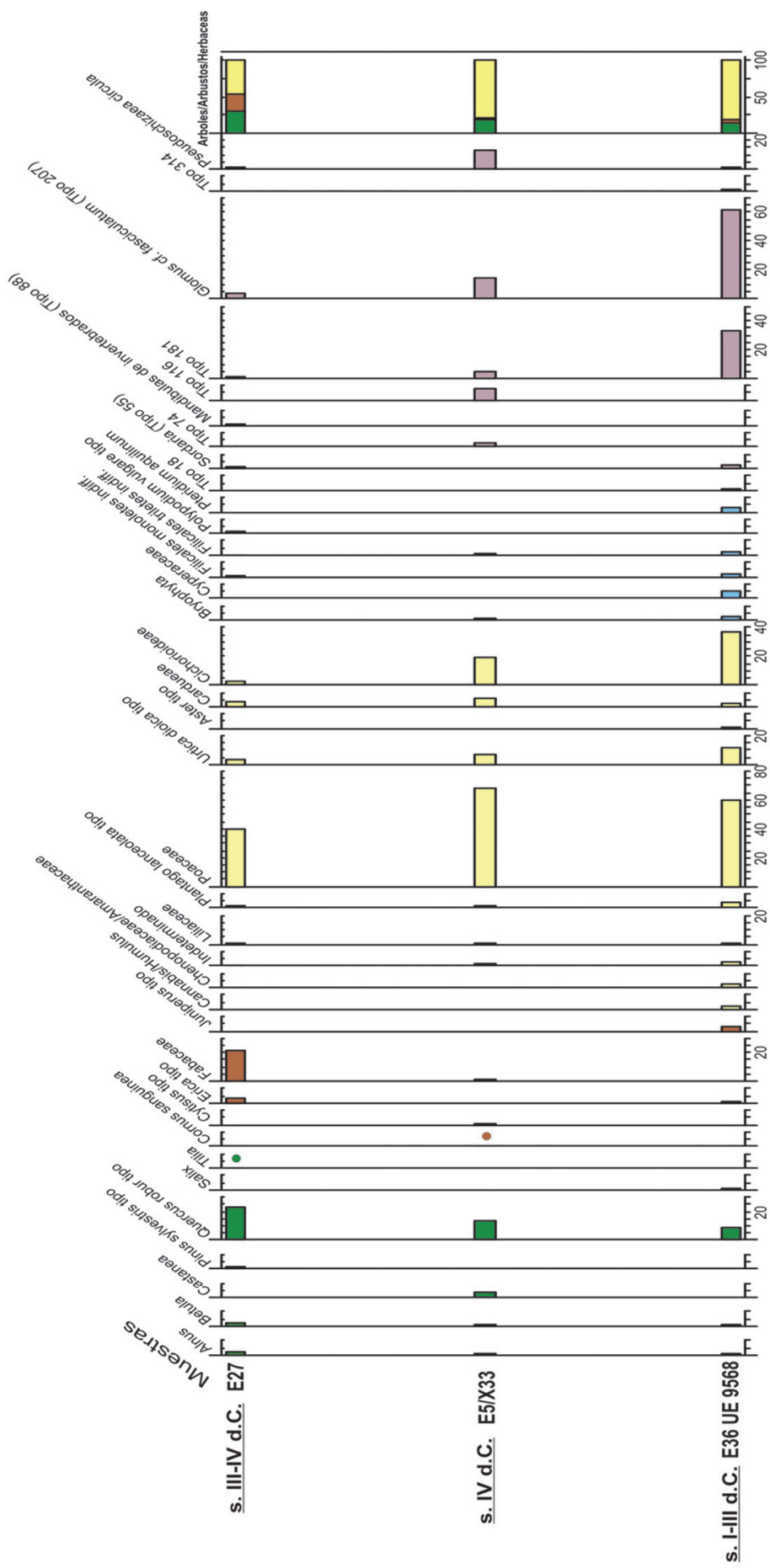

Figura 3. Resultados palinológicos del granero de la villa romana de Veranes (Gijón). Muestras E27, E36 y espacio E5 (López Sáez 2012, 163, fig. 74). 
cultivo del nogal (Juglans) y apareciendo el castaño (Castanea). De igual modo, se testimonia una disminución importante de los niveles de polen de cereal (Cerealia). En el marco de las investigaciones palinológicas realizadas en la villa de Veranes que aquí se presentan se decidió, asimismo, llevar a cabo estudios polínicos a alta resolución en un depósito turboso situado en las inmediaciones (Monte Areo), con objeto de poder comparar dos contextos sedimentarios diferentes (arqueológico, natural) y establecer la consiguiente correlación, cronológica y paleoambiental, entre un registro continuo (turbera de Monte Areo) y otro fraccionario (yacimiento arqueológico de Veranes).

Tan interesantes como los estudios palinológicos de Veranes son los análisis de macrorrestos realizados en Houten-Tielland (Utrecht, Holanda); en este emplazamiento se percibe que, a comienzos del periodo romano, los agricultores decidieron sembrar especies más aptas para cultivos de verano, como la Avena sativa, el Hordeum vulgare var. vulgare y el Triticum dicoccum que, además, aceptan suelos menos favorables. En cambio, no se cultivaron en este área ni Triticum aestivum ni Triticum spelta.$^{14}$

La interpretación conjunta de los datos aportados por los análisis arqueobotánicos, que nos ofrecen mayor información sobre el estudio de las prácticas agrícolas, es todavía exigua y las conclusiones a las que podemos llegar son, sin duda, parciales. No obstante, trataremos de aproximarnos a la problemática arqueobotánica en relación al estudio de los graneros romanos.

El principal problema que encontramos es la ausencia de la aplicación de este tipo de análisis al estudio de los graneros, siendo prácticamente nulos por el momento en las regiones meridionales mediterráneas y muy escasos en el caso de las islas británicas. También encontramos una rala información en las memorias de excavación, hecho que comporta tanto la indefinición de las especies de trigo almacenadas ${ }^{15}$ como del modo concreto de almacenaje del cereal, pues no se determina si se conservan en espiga, en espiguilla o en grano; afortunadamente, sí se suele mencionar la presencia de insectos o síntomas de germinación. En otros casos, solamente conocemos datos muy genéricos, como los restos de cultivos; así sucede con el granero de Wantage, Mill Street (Inglaterra). ${ }^{16}$

\section{3. ¿Qué especies de grano se almacenaron en los horrea rurales romanos?}

Generalmente los macrorrestos vegetales que aparecen en relación con los horrea romanos aparecen preservados en estado carbonizado. En medios húmedos, donde las condiciones de preservación son óptimas, podrían aparecer sin carbonizar, pero por el momento no contamos con este tipo de restos.

14 Kooistra 1996, 291-368.

15 Así sucede en el estudio de los graneros de Estrées-Sant-Denis (Francia; Matterne 2002), Les Mureaux (Francia; CAG 78. Les Yvelines: 253-259), Gilly (Francia; Barthélémy 1986), Gourdon (Francia; Buchet 2001), en la torre-silo de Mageroy (Bélgica; Halbardier - Gaimant 1989), en el granero de madera de Les Râpettes en Marly (Fribourg, Suiza; Haldimann 1985), Oberndorf-Bochingen (Alemania; Csysz 1990; Sommer 2001 y 2002), Selongey (Francia; Olivier - Ruellet 1990, 78) y Hauts de Clauwiers (Francia; Révillion et alii 1994; Révillion - Bouche 2003).

16 Holbrook - Thomas 1994 y 1996. 
Las especies de cereales almacenadas en los graneros rurales romanos fueron variadas. En escasos horrea -como el de Maurepas (Francia) $-{ }^{17}$ se ha localizado aisladamente trigo desnudo, mientras que en otros se han hallado vestidos, es decir, con espiguillas o con su envoltura: así ha sucedido en la despensa de Bliesbruck (Francia), donde se halló grano espelta, ${ }^{18}$ en el granero de Borg (Alemania), en el que se reconoció Hordeum vulgare vulgare y Triticum dicoccum ${ }^{19} \mathrm{y}$ en el granero de Laschamps (Francia), en el que se documentó trigo espelta, Triticum dicocum y avena. ${ }^{20}$ Finalmente, en otros graneros se han encontrado mezclados trigos desnudos y vestidos, como en los horrea de Wendens Ambo (Inglaterra), ${ }^{21}$ Bad Dürkheim-Ungstein, ${ }^{22}$ Bad Rappenau-Babstadt (Alemania), ${ }^{23}$ Emptinne-Champion (Bélgica), ${ }^{24}$ Houdan (Francia), ${ }^{25}$ Maasbracht (Países Bajos) ${ }^{26}$ y Mont Beuvray (Francia). ${ }^{27}$

\section{No sólo grano: otros alimentos almacenados en los horrea}

En algunos almacenes se conservaron, además de las diferentes especies de trigo, otros alimentos, como las leguminosas, posiblemente de manera diferenciada. En este periodo la producción de leguminosas se multiplicó y se almacenó junto a los cereales. Así parecen demostrarlo las lentejas conservadas con cebada en el granero de Marollessur-seine (Francia $)^{28}$ o en el de la aglomeración secundaria de Crest (Francia); en el otro almacén de Crest se localizaron, de modo conjunto, trigo desnudo, cebada, habas y frutas..$^{29}$ En este tipo de almacenaje mixto también aparecen otras legumbres, como los guisantes, detectados en Jülich-Kirchberg, WW 112 (Alemania); ${ }^{30}$ por su parte, en Alle, Les Aiges (Jura, Suiza) $)^{31}$ y Villiers-le-Sec (Francia) ${ }^{32}$ se han documentado trigo, avena, cebada, guisantes y habas, y en Voerendaal (Limburg, Países Bajos) ${ }^{33}$ varios tipos de trigo junto con guisantes y habas.

La heterogeneidad de cultivos que caracteriza a la época romana se ve también reflejada en los hallazgos de los graneros, en los que se constatan especies que debieron ser introducidas o importadas, posiblemente relacionadas con la arboricultura y el comercio. ${ }^{34}$ Son cultivos que probablemente se potenciaron en este periodo y conocieron un gran impulso en época clásica. Las especies frutales pudieron ser transportadas a

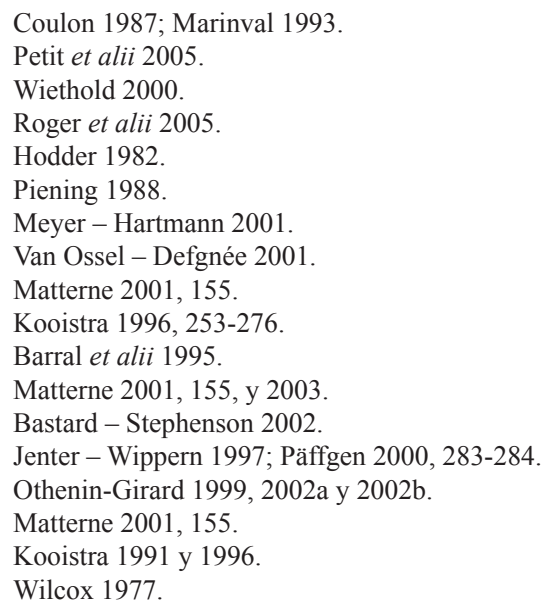


grandes distancias empleando diferentes técnicas de conserva, bien en forma de pasa seca (higos, uvas, ciruelas...), bien en ánforas con líquidos (aceituna, melocotón...).

Este hecho es evidente en el horreum de Alise-Sainte-Reine (Francia), cuyas muestras testimonian el almacenaje de Triticum spelta, Triticum dicoccum, cebada limpia, mijo común, avena y Triticum aestivum/durum, además de pipas de uva, algunas semillas de altramuz blanco (Lupinus albus var. albus), melón (Cucumis melo) y calabaza (Lagenaria siceraria).$^{35}$ Otro tanto ocurre en el de la villa romana de Biberist-Spitalhof (Suiza) en el que, además de cebada y trigo escanda, se hallaron restos de frutos de hueso (ciruelas, ciruelas, cerezas, etc.), ${ }^{36} \mathrm{o}$ en el de Lournand (Francia), donde se hallaron pipas de uva carbonizadas. ${ }^{37}$

\section{5. ¿Cómo se almacenó el cereal?}

En graneros con el pavimento sobreelevado la forma de almacenar el cereal consiste, generalmente, en verter a granel las semillas en espigas en la superficie del suelo, aunque se trate de cereales desnudos o vestidos, cebada o trigo. Los análisis realizados demuestran que se almacenaba el cereal a granel en los horrea del campamento militar de South Shields, ${ }^{38}$ Valkenburg, ${ }^{39}$ así como en los urbanos de Alcester, ${ }^{40}$ Londres, ${ }^{41}$ Amiens ${ }^{42}$ y en el granero de la villa de Voerendaal. ${ }^{43}$ Las fuentes clásicas mencionan la construcción de dichos espacios para separar los tipos de granos ${ }^{44}$ y el estudio de los restos de semillas aparecidos en el interior del horreum de Amiens confirma una cierta organización interna y la separación de productos en la cámara de almacenamiento. ${ }^{45}$

La conservación del grano aún vestido aumenta la protección contra los riesgos derivados de la humedad, a menudo responsable de una germinación anticipada, o de su degradación por la acción de los insectos. Las vainas del grano, además, garantizan la limpieza del mismo durante todo el período de almacenamiento; por ello, Plinio $^{46}$ recomienda conservar el grano en espiga. Se guardaron mayoritariamente con glumas en la despensa de la villa romana de Alise-Sainte-Reine (Francia) ${ }^{47}$ y también en espiga ha aparecido el trigo almacenado en la despensa de Houdan (Francia). ${ }^{48}$ Aunque no son muchos los macrorrestos analizados, podemos determinar que los granos vestidos son a menudo almacenados en forma de espiga o espiguilla tras un primer cribado o trillado, pues el desgrano no se puede llevar a cabo cuando se trata de grandes volúmenes de cereal, ya que esta tarea monopolizaría

\footnotetext{
Wiethold 2003.

Schucany 2006.

Ferdière 1988, 76.

Van der Veen 1988.

Pals et alii 1989.

Booth 1989.

Straker 1984.

Matterne et al. 1998.

Kooistra 1996.

Colum. 1.6.9-24.

Matterne et alii 1998.

Plin. HN 18.73.306.

Wiethold 2003.

Matterne 2001, 155.
} 
todo el trabajo de la cosecha. En cambio, el grano desnudo se suele almacenar o limpiar generalmente en los establecimientos rurales, como confirman los análisis de macrorrestos.

A pesar de las ventajas que comportaba almacenar el cereal en espiguillas -que, como ya se adelantó, aísla mejor al grano de la humedad y reduce los riesgos de germinación- en época romana también se optó por guardarlo completamente limpio. El hecho de que se almacenase de este modo nos podría indicar que iba a ser consumido muy posiblemente en el lugar, lo que nos informa, a su vez, sobre un alto grado de autoconsumo, de modo que donde se producía, se consumía. Este parece ser el caso del horreum de Wendens Ambo (Inglaterra), donde apareció un secadero de grano en el que se procesaría el Triticum aestivum, el Triticum spelta y el Triticum Diccocum conservados. ${ }^{49}$

De otro lado, determinados estudios han permitido documentar las malas hierbas que acompañan al grano en el interior de los almacenes, hierbas que corresponden a los tipos encontrados en las cercanías a cultivos y cosechas. Así, en el granero de Emptinne-Champion (Bélgica), se ha registrado la presencia de bromo (Bromus sp.), amor de hortelano (Galium aparine), polígono trepador (Polygonum Convolvulus), y hierba pejiguera (Polygonum lapathifolium), además de cenizo (Chenopodium album), llantén lanceolado (Plantago lanceolata) y acedera menor (Rumex acetosella) $;{ }^{50}$ interesante es también la documentación, en este mismo granero, de alfalfa (Medicago sativa) y de arveja (Vicia sativa), que se cultivaban como forraje. Del mismo modo, se han detectado Medicago lupulina y Raphanus raphanistrum entre los diferentes tipos de trigo encontrados en la despensa de Maasbracht (Países Bajos). ${ }^{51}$ También en los graneros de Voerendaal (Limburg, Países Bajos) aparecieron malas hierbas; a pesar de que el grano fue trillado y limpiado antes de ser almacenado en el horreum, la proporción de malas hierbas y paja era alta, siendo predominante la Agrostemma githago, el Bromus secalinus y el Scandix Pecten-veneris. ${ }^{52}$ Los granos de Apera spica-venti y las semillas de Papaver dubium/p. rhoeas, aparecidos también en este mismo granero, son residuos del trillado.

\section{Las consecuencias de una mala conservación o un almacenaje prolongado: la germinación}

Los problemas de germinación de los granos almacenados parecen iniciarse en época romana, con la construcción de los grandes almacenes y la conservación de un volumen enorme de cereal, a largo plazo, asociado a una política de redistribución y de circulación a larga distancia que comportó grandes problemas y pérdida de la mies.

La germinación puede, o bien ser controlada, para el malteado y fabricación de la cerveza, o bien ser accidental, ligada a malas condiciones ambientales, con una humedad relativa excesiva, que supere o se encuentre entre 20 y $25 \%$. La germinación

\footnotetext{
Hodder 1982.

Van Ossel - Defgnée 2001.

Kooistra 1996, 253-276.

Kooistra 1991 y 1996.
} 
controlada se ha podido constatar en la despensa en sótano de Bliesbruck (Francia), donde se halló grano de espelta, aún envuelto en sus glumas, que empezó a germinar en el momento de su carbonización, lo que sugiere la hipótesis de que servía para la producción de cerveza. ${ }^{53}$

Es importante recordar que la conservación del grano aún vestido aumenta la protección contra el medio húmedo, a menudo responsable de una germinación anticipada o de su degradación por la acción de los insectos. Gran parte del trigo encontrado en el granero de Mont Beuvray (Francia) también se hallaba quemado y en estado de germinación. ${ }^{54}$ Este es un factor que se debe tener en cuenta a la hora de plantear cálculos de capacidad, pues tanto la altura de la acumulación de grano como su disposición influyen en su posterior conservación.

\section{La Teoría del Escepticismo Cuantitativo (TEC)}

A pesar de los datos aportados por los análisis arqueobotánicos y la información obtenida a partir de la realización de excavaciones, cada vez más exhaustivas, aún estamos a las puertas de determinar tanto la capacidad de los graneros romanos, como el potencial agrícola de los centros de producción. A día de hoy podemos cuestionar las diferentes propuestas de capacidad de los horrea romanos en función de los siguientes factores:

1. En la mayoría de los graneros desconocemos el tipo de cereal que fue conservado. Esta información es primordial, pues el peso de cada especie es diferente $^{55}$ (Fig. 4).

2. En los horrea no sólo se conservó grano, sino también otros productos, tal y como la arqueobotánica nos informa y hemos señalado en páginas precedentes.

3. Desconocemos en líneas generales el modo de almacenaje: a granel, en sacos o en cajones de madera, etc.

4. Igualmente, en buena parte de los casos ignoramos el modo concreto de conservación: en espigas o limpio.

5. Se puede plantear un cálculo teórico de un granero repleto, pero los almacenes eran continuamente vaciados en cada cosecha, en función de las necesidades de sus propietarios. El granero nunca nos podrá ofrecer una estimación de la potencialidad agrícola de un centro rural, pues la mayor parte de la cosecha estaba destinada a la venta, a la redistribución y comercialización casi inmediata. Al propietario de un establecimiento rural no le interesaba, desde el punto de vista económico, conservar un producto tan perecedero como el grano.

6. Los estudios arqueobotánicos indican que no toda la superficie del pavimento estaría cubierta de grano, pues era necesario habilitar espacios de circulación y ventilación para evitar la germinación del grano; además, es muy posible

\footnotetext{
Petit et alii 2005.

Barral et alii 1995.

Gentry 1976, 25
} 


\begin{tabular}{|c|c|c|}
\hline Tipo de grano & $\mathbf{K g} / \mathbf{m}^{\mathbf{3}}$ & $\mathbf{M}^{3} /$ tonelada \\
\hline Trigo & 785 & 1,3 \\
\hline Cebada & 705 & 1,4 \\
\hline Avena & 513 & 1,9 \\
\hline Centeno & 705 & 1,4 \\
\hline
\end{tabular}

Figura 4. Peso de los principales cereales almacenados en los horrea rurales romanos según la información de Gentry $(1976,25)$.

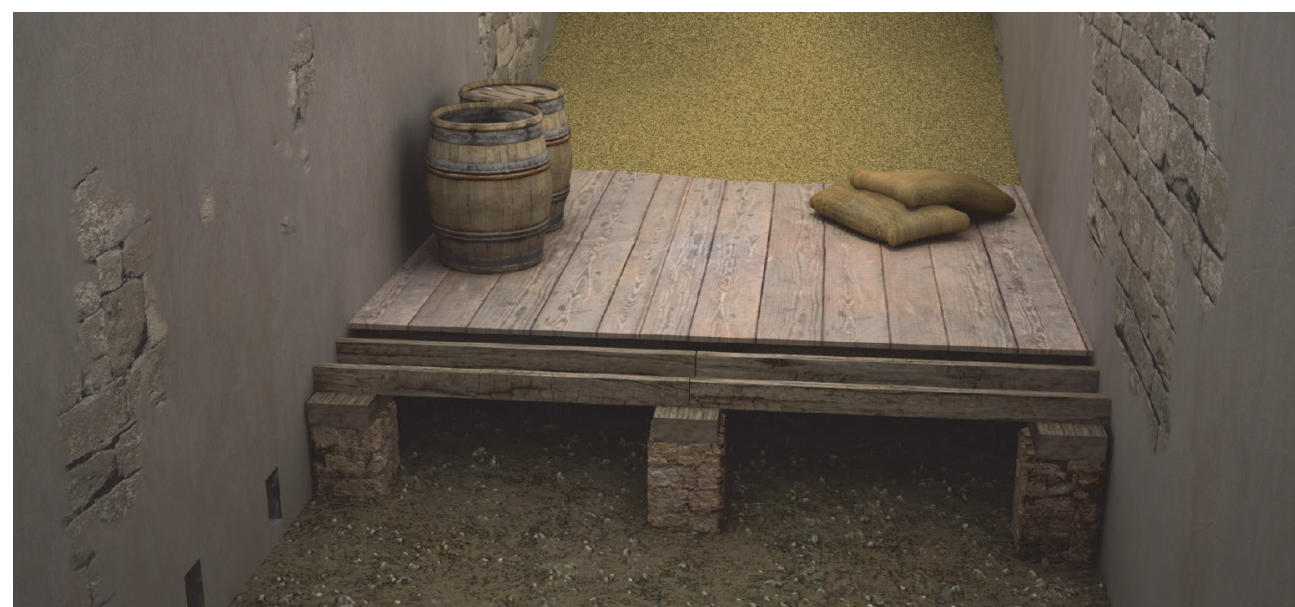

Figura 5. Reconstrucción del tabulatum y apoyos del granero de la villa romana de Veranes, Gijón (Fernández Ochoa et alii 2012, 134, fig. 57).

que en el interior de los horrea existiesen diferentes compartimentos para diferenciar los tipos de grano.

7. Es necesario calcular la resistencia de los tabulata o sobrados del granero, un cómputo difícil de estimar si desconocemos el material con el que fueron construidos. En la mayoría de los graneros estos sobrados son de madera, pero el tipo condiciona la resistencia del suelo (Fig. 5).

8. No existe acuerdo entre los especialistas al respecto de la altura que el amontonamiento del grano debía alcanzar para que este se mantuviera en buenas condiciones de conservación (Fig. 6).

Gentry plantea que podría almacenarse hasta una altura de $2 \mathrm{~m} ;{ }^{56}$ en cambio, Manning señala que se conservaban hasta $1,5 \mathrm{~m} .{ }^{57}$ En opinión de Sigaut no sería posible almacenar el grano hasta una altura de más de $1 \mathrm{~m}$ para un amon- 


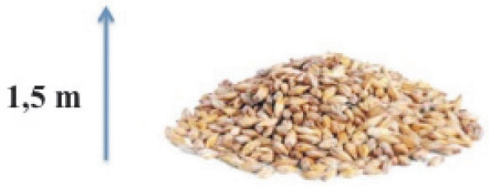

Manning 1975

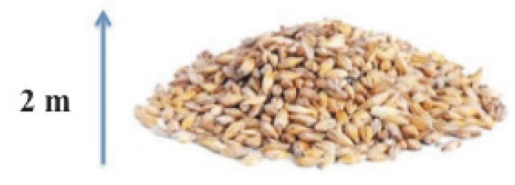

Gentry 1976

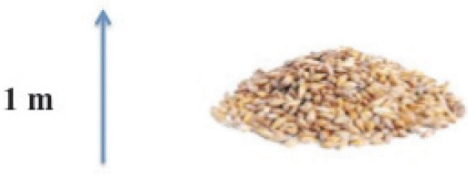

Sigaut 1981

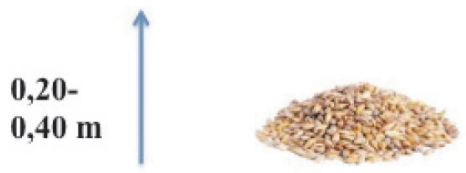

Matterne 1991

Figura 6. Diferentes propuestas de altura del amontonamiento del grano.

tonamiento de unos $2 \mathrm{~m}$ de extensión en su base. ${ }^{58}$ Aunque recientes investigaciones determinan que el espesor medio de la capa de cereal debe ser de entre 20 y $40 \mathrm{~cm}^{59}$ para mantener las condiciones óptimas de conservación del cereal y evitar la germinación del grano, es cierto que el monto total de grano almacenado a esta altura resulta, cuando menos, escaso. Sin duda, la altura del amontonamiento dependería del tiempo de almacenaje y de la posibilidad de acceder a las distintas partes de la cámara para voltear el grano y evitar, así, la germinación o infestación del cereal situado en la parte inferior, más expuesto al aumento de la temperatura.

9. No sabemos cuánto tiempo se mantuvo el cereal en el granero. Si consideramos la información textual y arqueológica, podemos concluir que el cereal almacenado no es representativo de la capacidad agrícola de un centro rural romano, pues un horreum no necesariamente debe mantener el grano durante un año. ${ }^{60}$

A partir de esta información, nos vemos obligados a revisar los estudios de cuantificación de la capacidad máxima de grano almacenado en los horrea excavados, así como a reconsiderar los datos referidos a la explotación agrícola de un territorio y a la cantidad de cereal estimado para el consumo directo y la exportación. En definitiva, planteamos la necesidad de cuestionar o relativizar las propuestas de capacidad ofrecidas hasta el momento, como las publicadas por Gentry o Manning para el estudio de los graneros militares, hasta que el estudio multidisciplinar que aúne los principios físicos de resistencia de los tabulata y de preservación del grano nos permita abordar esta problemática de la forma más acertada posible.

\footnotetext{
Sigaut 1981, 168.

Matterne et alii 1998.

Salido 2017a, 81-82.
} 
En conclusión, nos parece poco correcto proponer más datos sobre cuantificación si estos no responden a la realidad (Teoría del Escepticismo Cuantitativo). Por ello, abogamos por tener en cuenta más el valor cualitativo que el cuantitativo-comparativo del almacenaje de los horrea para confrontar la capacidad de los diferentes graneros. De este modo, podremos establecer una correlación del volumen de los diferentes horrea con el fin de indicar los edificios que presentaban una mayor o menor capacidad y, así, determinar los centros de llegada, recogida y aprovisionamiento; ello, a su vez, nos permitiría llegar a conclusiones sobre el procedimiento y envío de grano, las redes comerciales y las técnicas de aprovisionamiento del cereal en época romana. Esperemos que, en un futuro cercano y con la ayuda de las nuevas tecnologías, estudios físicos nos permitan resolver o solventar en parte este problema arqueológico.

\section{Referencias bibliográficas}

Barral, P. - Beck, P. - Bernal, J. (1995): "Les fouilles du Mont Beuvray, Nièvre, Saône-etLoire: rapport biennal 1992-1993”, Revue archéologique de l'Est et du Centre-Est 46/2, $\mathrm{n}^{\circ} 168,217-293$.

Barthélémy, H. (1986): “Un site gallo-romain alpin: Gilly (Savoie)”, Revue archéologique de Narbonnaise 19, 211-244.

Bastard, V. - Stephenson, P. (2002): "Entre structures agraires, sanctuaire et station routière: le site de Bourbousson 3 à Crest (Drôme)", [en] T. Janin (dir.), Archéologie du TGV Méditerranée. Fiches de Synthèse III: Antiquité, Moyen âge, Époque moderne (=Monographies d'Archéologie Méditerranéenne 10), Lattes, 687-698.

Boetto, G. - Bukowiecki, E. - Monteix, N. - Rousse, C. (2016): "Les Grandi Horrea d'Ostie", B. Marin - C. Virlouvet (eds.), Entrepôts et trafics annonaires en Méditerranée. AntiquitéTemps Modernes (=Collection de l'École française de Rome 522), Roma, 177-226.

Booth, P. M. (1989): "Roman store buildings at Alcester", Transactions Birmingham and Warwickshire Archaeological Society 94, 63-106.

Brinkkemper, O.

(1991): Wetland farming in the area to the south of the Meuse estuary during the Iron-Age and Roman period. An environmental and paleo-economic reconstruction (=Analecta praehistorica Leidensia 24), Leiden.

(1995): "A native settlement from the Roman period near Rockanje", Berichten van de Rijksdienst voor het Oudheidkundig Bodemonderzoek 41, 123-171.

Buchet, L. (2001): 'Le village de la 'Bergerie du Montet' (Gourdon) de la Protohistorie à la fin de l'Antiquité", [en] P. Arnaud - M. Gazenbeek (eds.), Habitat rural antique dans les Alpes-Maritimes (Valbonne, 1999), Antibes, 33-57.

Coulon, G. (1987): "L'implantation gallo-romaine en Bas-Berry. Présentation de quelques découvertes récentes et de sites méconnus", Revue de l'Académie du Centre 1987, 39-58.

Csysz, W. (1990): “Ausgrabungen in einem römischen Gutshof bei Oberndorf a. Lech”, Das archäologische Jahr in Bayern, 133-140.

Ferdière, A. (1988): Les Campagnes en Gaule romaine. Tome 1: Les Hommes et l'environnement en Gaule rurale (52 av. J.-C.-486 ap. J.-C.). Tome 2: Les Techniques et les productions rurales en Gaule (52 av. J.-C.-486 ap. J.-C.), Paris.

Fernández Ochoa, C. - Gil Sendino, F. - Salido Domínguez, J. - Zarzalejos Prieto, M. (eds.), (2012): El horreum de la villa romana de Veranes (Gijón, Asturias). Primer testimonio material de los hórreos de Asturias, Madrid. 
Gentry, A. P. (1976): Roman military stone-built granaries in Britain (=BAR International Series 32), Oxford.

Halbardier, B. - Gaimant, P. (1989): "Villa gallo-romaine de Mageroy (Habay-la-VieilleHabay, Lux.)", Vie archéologique 33-34, 27-30.

Haldimann, M. A. (1985): "Marly (Sarine), Les Râpettes", Archéologie fribourgeoise. Chronique archéologique 1985, 34-57.

Hermansen, G. (1982): Ostia. Aspects of Roman City Life, Alberta.

Hodder, I. (1982): Wendens Ambo. The Excavations of an Iron Age and Romano-British Settlement, London.

Holbrook, N. - Thomas, A. (1994): "Wantage, Mill Street (SU 395881)", South Midlands Archaeology 24, 31.

Jenter, S. - Wippern, J. M. (1997): "Prospektionsergebnisse von einer römischen villa rustica bei Kirchberg”, Archäologie im Rheinland 1996, 177-180.

Kooistra, L. I.

(1991): "Arable Farming in the Heyday of the Roman Villa at Voerendaal (Limburg, the Netherlands)", [en] E. Hajnalová (ed.), Paleoethnobotany and Archaeology: International Work-Group for Paleoethnobotany 8th Simposium (Nitra-Nové Vozokany, 1989), (=Acta Interdisciplinaria Archaeologica 7), Nitra, 165-175.

(1996): Borderland Farming. Possibilities and Limitations of Farming in the Roman Period and Early Middle Ages between the Rhine and Meuse, Amersfoort.

Küster, H. (ed.), (1988): Der prähistorische Mensch und seine Umwelt (=Forsch. u. Ber zur Vor-u. Frühgesch. Baden-Württemberg 30), Stuttgart.

López Merino, L. - Martínez Cortizas, A. - López Sáez, J. A. (2010): “Early agriculture and palaeoenvironmental history in the North of the Iberian Peninsula: a multi-proxy analysis of the Monte Areo mire (Asturias, Spain)", Journal of Archaeological Science 37, 19781988 (http://dx.doi.org/ 10.1016/j.jas.2010.03.003).

López Sáez, J. A. (2012): “Análisis polínicos”, [en] Fernández Ochoa et alii (eds.), 2012, 157-166.

Manning, W. H. (1975): "Roman military timber granaries in Britain", Saalburg Jahrbuch 32, 105-129.

Marinval, P. (1993): "Deux amas de grains de blé carbonisés du Haut-Empire romain provenant de Pithiviers-le-Vieil (Loiret) et de Liniez (Indre)", Revue Archéologique du Loiret $18,105-119$.

Matterne, V.

(2001): Agriculture et alimentation végétale durant l'âge du Fer et l'époque gallo-romaine en France septentrionale, Montagnac.

(2002): "Les fruits et les graines provenant de quelques fosses, fossés et dépotoirs du site d'Estrées-Saint-Denis (Oise)", [en] P. Querel - G.-P. Woimant (eds.), Le site antique d'Estrées-Saint-Denis (Oise). Sanctuaire et habitat, une agglomération secondaire? (=Revue Archeologique de Picardie 2002/3-4), Amiens, 367-370.

Matterne, V. - Yvinec, J.-H. - Gemehl, D. - Riquier, Ch. (1998): “Stockage de plantes alimentaires et infestation par les insectes dans un grenier incendié de la fin du $\mathrm{II}^{\mathrm{e}}$ siècle après J.-C. à Amiens (Somme)", Revue Archeologique de Picardie 3-4, 93122.

Meyer, F. J. - Hartmann, H.-H. (2001): "Ein horreum in der villa rustica in Bad RappenauBabstadt, Kreis Heilbronn", Arch. Ausgr. Baden-Württemberg 2001, 127-130.

Olivier, A. - Ruellet, A. (1990): “Selongey. La villa”, [en] Archéologie de Bourgogne. 1, La Côte-d'Or, Dijon, 78. 
Othenin-Girard, B.

(1999): “Alle JU, Les Aiges", Jahrbuch der Schweizerischen Gesellschaft für Ur- und Frühgeschichte 82, 277.

(2002a): Greniers gallo-romains et vestiges funéraires du Bronze final à Alle, Les Aiges (Jura, Suisse). Fouilles 2001 (=Archéologie et Transjurane 95), Porrentruy.

(2002b): “Alle JU, Les Aiges”, Jahrbuch der Schweizerischen Gesellschaft für Ur- und Frühgeschichte 85, 306.

Päffgen, B. (2000): “Villa rustica und Burgus auf dem Steinacker in Jülich-Kirchberg”, [en] H. G. Horn (dir.), Fundort Nordrhein-Westfalen. Millionen Jahre Geschichte (=Schriften zur Bodendenkmalpfiege in Nordrhein-Westfalen 5), Mainz, 283-284.

Pals, J. P. - Beemster, V. - Noordam, A. (1989): "Plant remains from the Roman castellum Praetorium Agrippinae near Valkenburg (prov. of Zuid-Holland), Archäobotanik", Dissertationes Botanicae 133, 117-134.

Petit, J.-P. - Schaub, J. - Brunella, Ph. (2005): Bliesbruck-Reinheim: Celtes et Gallo-Romains en Moselle et en Sarre, Paris.

Piening, U. (1988): "Verkohlte P anzenreste aus zwei römischen Gutshöfen bei Bad Dürkheim (Pfalz)", [en] Küster (ed.), 1988, 325-340.

Pilon, F.

(2003): 'Production de fausse monnaie en milieu rural: la villa du 'Bois du Châtel' à Vieux-Champagne”, Revue Archeologique de Picardie 2003/1-2, 177-183.

(2005): 'La villa gallo-romaine du 'Bois du Châtel' à Vieux-Champagne (Seine et Marne)", L'archéologue 78, 34-37.

Révillion, S. - Bouche, K. (2003): “Architecture de terre et de bois dans la vallée de la haute Deûle, de la fin de l'Antiquité au début de l'époque mérovingienne: apports des gisements du 'Luyot', du 'Haut de Clauwiers' et de 'l'Epinette' à Seclin (Nord)', Revue du Nord 85, 113-124.

Révillion, S. - Bouche, K. - Wozny, L. (1994): "La partie agricole d'une grande exploitation rurale d'époque romaine: le gisement du 'Haut de Clauwiers', Seclin (Nord)", Revue du Nord 76/308, 99-146.

Roger, J. - Bet, Ph. - Simon, L. - Garraud, N. - Poirier, P. (2005): "L'établissement rural gallo-romain de Laschamps à Parsac (Creuse)", Aquitania 21, 151-186.

Salido Domínguez, J.

(2003-2004) "La documentación literaria aplicada al registro arqueológico: las técnicas de construcción de los graneros romanos rurales", Espacio, Tiempo y Forma. Serie I: Prehistoria y Arqueología 16-17, 463-478 (https://doi.org/10.5944/etfi.16-17.2003.4765). (2008a): "La investigación sobre los horrea de época romana: balance historiográfico y perspectivas de futuro", CUPAUAM 34, 105-124 (http://dx.doi.org/10.15366/cupauam2008.34.005).

(2008b): "Los sistemas de almacenamiento y conservación de grano en las villae hispanorromanas", [en] C. Fernández Ochoa - V. García-Entero - F. Gil Sendino (eds.), Las villae tardorromanas en el Occidente del Imperio. Arquitectura y función. IV Coloquio Internacional de Arqueología de Gijón, Gijón, 693-706.

(2011a): Horrea Militaria. El aprovisionamiento de grano en el Occidente del Imperio romano (=Anejos de Gladius 14), Madrid.

(2011b): "El almacenamiento de cereal en los establecimientos rurales hispanorromanos", [en] J. Arce - B. Gofaux (eds.), Horrea d'Hispanie et de la Méditerranée romaine, Madrid, 127-142. (2013): "El transporte marítimo de grano en época romana. Problemática arqueológica", [en] R. Morais - H. Granja - Á. Morillo (eds.), O Irado Mar Atlantico. O naufrágio bético augustano de Esposende (Norte de Portugal), Braga, 139-178. 
(2017a): Arquitectura rural romana: graneros y almacenes en el Occidente del Imperio (=Archéologie et Histoire Romaine 35), Autun.

(2017b): "Contribution à l'etude du stockage des cereales: identification, caracterisation et interpretation des granaria et horrea de la peninsule Iberique", [en] Trément (dir.), 2017, 361-387.

Salido Domínguez, J. - Bustamante, M. (2014): Pistrina Hispaniae. Panaderías, Molinerías y el artesanado alimentario en la Hispania Romana (=Instrumentum 14), Montagnac.

Salido Domínguez, J. - Morais, R. (2013): "El horreum de la ciudad romana de Bracara Augusta (Braga, Portugal): funcionalidad, tipología y contexto", Sautuola 18, 199-212.

Salido Domínguez, J. - Neira, L. (2014): "Representaciones de horrea en la musivaria romana. Problemas para su identificación”, Lvcentvm 33, 147-170 (https://doi.org/10.14198/ LVCENTVM2014.33.12).

Schucany, C. (2006): Die römische Villa von Biberist-Spitalhof/SO. (Grabungen 1982, 1983, 1986-1989). Untersuchungen im Wirtschaftsteil und Überlegungen zum Umland (=Ausgrabungen und Forschungen 4), Zürich.

Sommer, C. S.

(2001): "Ein Grosses landwirtscha iches Nebengebäude in Oberndorf-Bochingen, Kreis Rottweil. Aspekte der römischen Architektur”, Arch. Ausgr. Baden-Württemberg 2000, 117-121.

(2002): "Hölzerne und andere Getreidespeicher in Oberndorf-Bochingen, Kreis Rottweil?", Arch. Ausgr. Baden-Württemberg 2001, 133-134.

Straker, V. (1984): "First and second century carbonised cereal grain from Roman London", [en] W. Van Zeist - W. A. Casparie (eds.), Plants and Ancient Man: Studies in palaeoethnobotany (Groningen, 1983), Rotterdam, 323-329.

Trément, F. (dir.), (2017): Produire, transformer et stocker dans les campagnes des Gaules romaines. Problèmes d'interprétation fonctionnelle et économique des bâtiments d'exploitation et des structures de production agro-pastorale (=Aquitania Suppl. 38), Bordeaux.

Van der Veen, M. (1988): “Carbonised grain from a Roman granary at South Shields, North East England”, [en] Küster (ed.), 1988, 353-365.

Van Ossel, P. - Defgnée, A. (2001): Champion, Hamois: une villa romaine chez les Condruses: archéologie, environnement et économie d'une exploitation agricole antique de la Moyenne Belgique (=Études et documents, Archéologie 7), Namur.

Vitelli, G. (1980): "Grain Storage and Urban Growth in Imperial Ostia: A Quantitative Study”, World Archaeology 12/1, 54-68 (http://dx.doi.org/10.1080/00438243.1980.9979780).

Wiethold, J.

(2000): "Kontinuität und Wandel in der landwirtschaftlichen Produktion und Nahrungsmittelversorgung zwischen Spätlatènezeit und gallo-römischer Epoche. Archäobotanische Analysen in der römischen Grossvillenanlage von Borg, Kreis Merzig-Wadern", [en] A. Haner - S. Von Schnurbein (eds.), Kelten, Germanen, Römer im Mittelgebirgsraum zwischen Luxemburg und Thüringen. Akten des internationalen Kolloquiums zum DFG-Schwerpunktprogramm "Romanisierung” (Trier, 1998), (=Kolloquien zur Vor- und Frühgeschichte 5), Bonn, 147-160.

(2003): "How to trace the 'Romanisation' of Central Gaule by archaeobotanical analysis?- Some considerations on new archaeobotanical results from France Centre-Est", [en] F. Favory - A. Vignot (eds.), Actualité de la recherche en histoire et archéologie agraires. Actes du Colloque AGER V, Beçanson, 269-282. 